\title{
In vitro activity of Pheroid vesicles containing antibiotics against Plasmodium falciparum
}

\author{
Lissinda H Du Plessis, Anel C van Niekerk, Marlene M Maritz and Awie F Kotzé
}

The macrolide antibiotics, erythromycin and azithromycin, have been studied for their potential antimalarial activity, but only modest activity has been demonstrated. In this study, we investigated the enhancement of the efficacy of these antibiotics in combination with a patented lipid-based drug delivery system, Pheroid technology. A chloroquine resistant strain of Plasmodium falciparum (RSA11) was incubated with the formulations for a prolonged incubation time (144h). Drug efficacy assays were conducted by analyzing the histidine-rich protein II levels of the parasites. The effects of azithromycin and erythromycin were compared with other antibiotics and standard antimalarial drugs. The poor water soluble nature of the drugs led to the formation of micro scale Pheroid vesicles with average particle sizes of $72.76 \pm 10.73 \mu \mathrm{m}$ for azithromycin and $100.62 \pm 29.27 \mu \mathrm{m}$ for erythromycin. The $\mathrm{IC}_{50}$ values of erythromycin and azithromycin alone and entrapped in Pheroid vesicles decreased statistically significant $(P \leqslant 0.05)$. Prolonged exposure was also statistically meaningful $(P \leqslant 0.05)$, although it seems that exposure need not exceed $96 \mathrm{~h}$. Pheroid vesicles also proved successful in decreasing the $\mathrm{IC}_{50}$ values of doxycycline, tetracycline and triclosan. Pheroid vesicles containing antibiotics could prove successful as a malaria treatment option.

The Journal of Antibiotics (2012) 65, 609-614; doi:10.1038/ja.2012.89; published online 24 October 2012

Keywords: azithromycin; erythromycin; HRP II ELISA assay; lipid-based drug delivery system; malaria; Pheroid vesicles; Plasmodium falciparum

\section{INTRODUCTION}

Parasitic diseases are a global problem and about $30 \%$ of the world's population has been infected by parasites at some stage. Malaria is one of the parasitic diseases that kill more people annually than any of the other parasitic infections combined. More than 2 billion people in 100 countries are at risk of being affected by malaria. There were at least 216 million cases of acute malaria reported in 2010 and about 655000 people died from malaria, $86 \%$ of which are children under 5 years of age. Sub-Saharan Africa is worst hit by the disease with $81 \%$ of the annual cases reported. ${ }^{1}$ Malaria is caused by protozoan parasites of the genus Plasmodium. There are four species of Plasmodium, which infect humans of which Plasmodium falciparum is most deadly. ${ }^{2}$ Despite major research efforts to date, no effective vaccine against $P$. falciparum malaria is currently market available. ${ }^{3}$ Antimalarial chemotherapy therefore remains the only weapon against malaria. The rapid development of resistance against existing antimalarial compounds poses a problem in the efforts to combat the disease. ${ }^{4} \mathrm{New}$ chemotherapy strategies are underway ${ }^{5-8}$ but is slow in delivering compounds on the market. In the efforts to address the problem two possible alternative strategies have emerged. One is the identification of novel molecular drug targets within the parasite., ${ }^{9,10}$ The other strategy is the development and use of novel drug delivery systems in order to improve the efficacy, specificity, tolerability and therapeutic index of existing antimalarial drugs. ${ }^{11-13}$

In recent years, some antibiotics have been studied for their antimalarial properties and are generally combined with antimalarials in an effort to overcome the global challenge of drug-resistant P. falciparum. ${ }^{14}$ Antibiotics, including tetracyclines, quinolines, macrolides and rifampin, have proved antimalarial action in vitro. ${ }^{15,16}$ Macrolides fundamentally consist of a macrocyclic lactone ring, which is where the groups' name originates from. ${ }^{17}$ Macrolide antibiotics have been used as antibacterial compounds for many years due to their versatility and broad spectrum of activity against gram-positive and gram-negative organisms. ${ }^{17,18}$ The prototype of the macrolide antibiotics is erythromycin, which was isolated in 1952 from Streptomyces erythreus. ${ }^{17}$ Newer macrolides include clarithromycin and azithromycin, semi-synthetic derivatives of erythromycin. The advantages of clarithromycin and azithromycin include a less frequent dosage regimen and both drugs seem to exude better tolerability. ${ }^{17,18}$ Erythromycin was the first macrolide to be introduced as a possible treatment and prophylactic agent in malaria. ${ }^{19}$ The in vitro and in vivo antimalarial activity of erythromycin was first described in 1972 and data suggest that further investigation into the antiplasmodial effect of this macrolide could hold apparent advantages in the treatment of malaria. ${ }^{20-22} \mathrm{~A}$ 
previous study has demonstrated that erythromycin in combination with antimalarials like chloroquine, quinine, and mefloquine have, respectively, shown synergism, potentiation and additive effects. ${ }^{14}$ However, a study conducted during the eighties in eastern Thailand, where chloroquine-resistance is especially prevalent and where quinine-resistance is slowly rising, concluded that high dosages erythromycin administered together with standard dosages of chloroquine and quinine, respectively, had no remarkable effect on the cure rate of falciparum infection. ${ }^{23}$ Azithromycin is a semisynthetic derivative of erythromycin and differs from the prototype by possessing a methyl-substituted nitrogen atom in the macrolide ring. This substitution contributes to various advantages above erythromycin, including a broader spectrum of activity, more encouraging pharmacodynamics, and a longer elimination halflife. ${ }^{24}$ Azithromycin is classified as the most potent macrolide antibiotic regarding malaria. ${ }^{25}$ An in vitro study displayed a 10 -fold greater potency of azithromycin over erythromycin against a chloroquine-resistant strain of $P$. falciparum. ${ }^{20}$ Azithromycin seems to illustrate better antimalarial activity by prolonging its exposure to malaria parasites. ${ }^{26}$ Azithromycin has not been licensed for use as an antimalarial drug yet, ${ }^{27}$ but this macrolide antibiotic has demonstrated activity against $P$. falciparum in vitro, ${ }^{16,28}$ in the murine malaria model ${ }^{29}$ and in randomized controlled clinical trials. ${ }^{30-32}$ Azithromycin is approved for use in children and is considered for use in combination with chloroquine during intermittent preventative treatment in pregnant women. ${ }^{33}$ However, the slow onset of antimalarial action of antibiotics excludes their use as single agents. ${ }^{28}$

The Pheroid drug delivery system is a patented colloidal system comprising of a unique submicron emulsion type formulation consisting mainly of plant and essential fatty acids. The Pheroid has been proven to entrap drugs with high efficiency and deliver these via various routes including nasal ${ }^{34}$ and orally. ${ }^{35}$ Pheroid have high entrapment efficacy of hydrophobic drugs like mefloquine and have good stability. ${ }^{12}$ Antibiotics have been successfully formulated with lipid-based drug delivery systems. One of the best examples is amphotericin B formulated in liposomes, which lead to the commercial product Ambisome. ${ }^{36}$ Ciprofloxacin and azithromycin has also been successfully formulated in liposomes and proved more successful than the drug alone against Mycobacterium avium infections. ${ }^{37}$

The aim of this study was (a) to investigate the in vitro antimalarial effects of erythromycin and azithromycin entrapped in the Pheroid vesicles against a chloroquine-resistant $P$. falciparum RSA 11 strain; (b) to evaluate whether the drugs entrapped in Pheroid vesicles could influence the time of the antimalarial action and (c) to compare the effects of azithromycin and erythromycin entrapped in Pheroid vesicles with standard antimalarial drugs and other antibiotics.

\section{MATERIALS AND METHODS}

RPMI-1640, D-(+)-glucose, HEPES, hypoxanthine, sodium bicarbonate, gentamycin, sodium chloride, sodium phosphate and potassium phosphate were obtained from Sigma Aldrich (St Louis, MO, USA). Albumax II and Nile Red were purchased from Life technologies, Invitrogen (Carlsbad, CA, USA). The special gas mixture, consisting of: $5 \%$ oxygen $\left(\mathrm{O}_{2}\right), 5 \%$ carbon dioxide $\left(\mathrm{CO}_{2}\right)$ and $90 \%$ nitrogen gas $\left(\mathrm{N}_{2}\right)$ and medicinal nitrous oxide was purchased from Afrox (Germiston, South Africa). Methanol, ethanol and dimethyl sulfoxide was obtained from Merck (Darmstadt, Germany) and the histidine-rich protein II (HRP II) ELISA kit (Malaria Ag CELISA) was obtained from Cellabs (Brookvale, NSW, Australia). Vitamin F ethyl ester was obtained from Kurt Richter Pharma (Berlin, Germany), Cremophor EL from BASF (Ludwigshafen, Germany) and D- $\alpha$-tocopherol from Chempure (Pretoria, South Africa).

\section{Formulations}

Azithromycin dihydrate was purchased from Iffect Chemphar (Hongkong, China). Erythromycin estolate was obtained from Lupid chemicals (Bangkok, Thailand). Chloroquine diphosphate, quinine, doxycycline and tetracycline were purchased from IPCA Laboratories (Mumbai, India). Triclosan (Irgasan) was purchased from Merck. Pheroid vesicles were prepared as described earlier. ${ }^{34}$ Test compounds were weighed and incorporated into the oil phase (4\%) of the Pheroid vesicles (co-formulating). Thereafter, the water phase (96\%) was added and the emulsion was left to shake for $12 \mathrm{~h}$. Test compounds were also entrapped in the diluted formulation after manufacturing (entrapment). In this method, Pheroid vesicles were prepared by adding the oil phase to the water phase and allowing the emulsion to shake for $12 \mathrm{~h}$, where after the dry product (erythromycin and azithromycin, respectively) was added to a 1:250 dilution of the Pheroid vesicles. Pheroid vesicles at 4 and $8 \%$ were used for optimization experiments. Control formulations consisted of the drugs alone dissolved in ethanol, dimethyl sulfoxide or double distilled water depending on the solubility of the drug. Pheroid vesicles without any drug entrapped also served as a control. In a separate experiment, chloroquine, quinine, doxycycline, tetracycline and triclosan were entrapped in the diluted Pheroid vesicles after manufacturing. The drug amounts differed from the macrolides, which resulted in slight variations in the entrapment method.

\section{Characterization of formulations}

Particle size was determined with a Malvern mastersizer (Malvern Instruments, Worcestershire, UK) by the method of laser diffraction. Adequate sample was added to $800 \mathrm{ml}$ of distilled water to obtain a laser obscuration between 10-20\% at a pump speed of 2500 r.p.m. Data were presented in histograms and the volume weighted means was used. The morphological features of Pheroid vesicles were assessed by both optical microscopic and confocal laser scanning microscopy. The optical microscopy images were taken with an inverted microscope (Nikon Eclipse TE-3000, Nikon Instruments, Melville, NY, USA). The confocal laser scanning microscopy images were taken with a Nikon D-Eclipse C1 confocal laser scanning microscope with a DXM 1200 digital camera with real time imaging and a medium $(10 \mathrm{pm})$ pinhole. A $60 \times 1.40$ ApoPlanar oil immersion objective was used. The microscope was equipped with a green krypton laser (wavelengths: excitation $488 \mathrm{~nm}$ and emission $515 \mathrm{~nm}$ ) and a red helium neon laser (wavelengths: excitation $505 \mathrm{~nm}$ and emission $564 \mathrm{~nm}$ ). Pheroids were labeled with the fluorophore Nile red $\left(1 \mathrm{mg} \mathrm{ml}^{-1}\right)$. The latter has an emission wavelength of between 640 and $650 \mathrm{~nm}$. Pheroids were placed on a microscope slide, covered with a glass cover slip and sealed with adhesive to prevent fluid loss. ${ }^{34}$

\section{Culture of $P$. falciparum}

The chloroquine-resistant $P$. falciparum strain RSA11 (South Africa) were maintained in culture as described previously ${ }^{38}$ with slight modifications. The parasites were kept at $37^{\circ} \mathrm{C}$ in a $5 \% \mathrm{O}_{2}, 5 \% \mathrm{CO}_{2}$ and $90 \% \mathrm{~N}_{2}$ enriched atmosphere. Parasites were cultured in RPMI 1640 medium containing $0.5 \%$ Albumax II, 0.6\% HEPES, $0.36 \mathrm{~mm}$ hypoxhantine, $5 \%$ sodium bicarbonate and $100 \mu \mathrm{g} \mathrm{ml}^{-1}$ gentamycin. Human red blood cells served as hosts.

\section{Drug efficacy assay}

Individual drugs in combination with Pheroid vesicles were tested, in duplicate, over an extended observation period of every $48 \mathrm{~h}$ for $144 \mathrm{~h}$. An initial parasitemia of $0.1 \%$ and hematocrit of $1.5 \%$ was used in experimenta procedures. A commercial test kit was used to determine histidine-rich protein II found only in P. falciparum. The kit uses two different monoclonal antibodies against $P$. falciparum HRP II. Before conducting an assay, samples were frozen after the incubation times and then thawed at room temperature in order to ensure complete hemolysis of red blood cells in each sample. After complete hemolysis was obtained, $100 \mu \mathrm{l}$ of each sample was transferred to the pre-coated (primary immunoglobulin $\mathrm{M}$ capturing antibody) ELISA plate. Following a 1-h incubation period at room temperature the plate was washed 

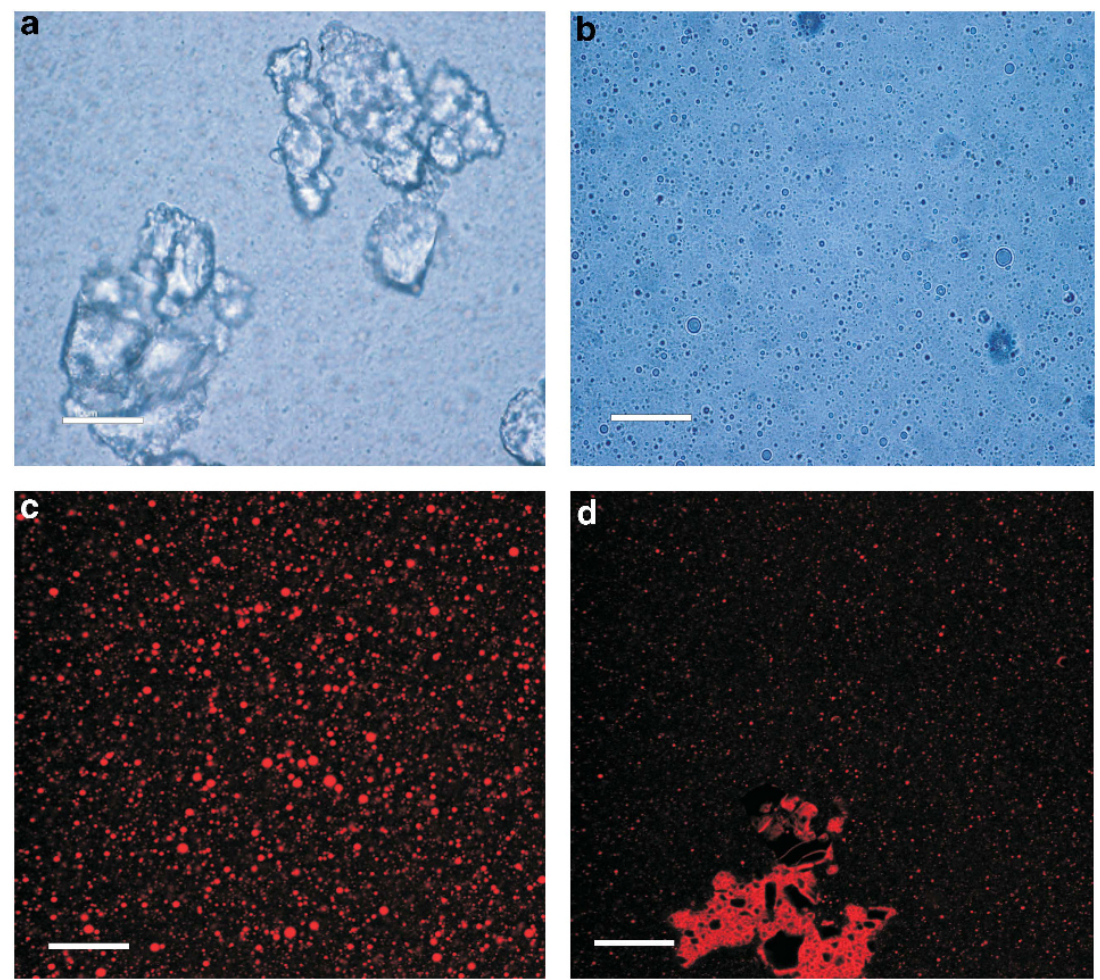

Figure 1 Light microscopy images of (a) 4\% Pheroid vesicle co-formulated with azithromycin, (b) $8 \%$ Pheroid vesicle formulation co-formulated with azithromycin and confocal microscopy images of (c) $4 \%$ Pheroid vesicle formulation with azithromycin entrapped in diluted Pheroid vesicles, (d) $8 \%$ Pheroid vesicle co-formulated with azithromycin. Scale bars represent $20 \mu \mathrm{m}$

four times. The conjugate containing the enzyme-secondary antibody (immunoglobulin G) was added to each sample and incubated for $1 \mathrm{~h}$ at room temperature in a humid chamber. The plate was once again washed four times and $100 \mu \mathrm{l}$ of the substrate containing tetramethyl benzidine was added to each sample. Samples were incubated, in the dark, for $14 \mathrm{~min}$. In the final step, $50 \mu \mathrm{l}$ of a stop solution containing $2 \mathrm{M}$ sulfuric acid was added to each sample. The plates were read with an ELX 800 Absorbance Microplate Reader with GEN5 data analysis software (BioTek, Winooski, VT, USA).

Quantification of this assay was based on the binding kinetics of a homogeneous recombinant histidine rich protein II (rHRP II). Levels of HRP II present in culture supernatant was then calibrated by implementing a standard graph created to allow measurement of rHRP II. Levels of HRP II present in unknown malaria experimental samples was measured in reference to the calibrated material, which served as the positive control. In order to evaluate the method a recombinant HRP II standard curve was generated through the serial dilution of the recombinant antigen (Recombinant Calibrator BM181, supplied with the kit). The recombinant was diluted in $100 \mu \mathrm{l}$ of $0.1 \mathrm{M}$ phosphate-buffered saline containing $0.01 \%$ Tween 20 (phosphate-buffered saline/T). A standard series $(0 ; 0.86 ; 1.72 ; 3.44 ; 6.80$; 13.75 and $27.50 \mathrm{ng} \mathrm{ml}^{-1}$ ) was prepared in a 96 -well plate. The standards were transferred to the pre-coated 96-well plate and the standard ELISA as described was performed. To determine the sensitivity of the method, especially at low parasitemia levels, a serial dilution of infected erythrocytes was made. The dilution ranged from 4 to $0.25 \%$ parasitemia at $1.5 \%$ hematocrit.

\section{Statistical analysis}

Results are expressed as mean \pm s.e.m. Statistical comparisons were obtained by GraphPadPrism 5.0 (GraphPad Software, San Diego, CA, USA) and Statistica (StatSoft, Tulsa, OK, USA). IC I0 $_{50}$ values were determined by means of non-linear regression. The best fit of the different data sets (Control vs Pheroid vesicles) was evaluated for statistical significant difference using the extra sum-of-squares $F$-test. The best fit value of the $\mathrm{IC}_{50}$ was chosen for comparison. The $\mathrm{IC}_{50}$ values was deemed significantly different when $P \leqslant 0.05$. Nonparametric analysis or log transformations were used for data that was not distributed normally.

\section{RESULTS AND DISCUSSION}

\section{Characterization of formulations}

Erythromycin and azithromycin are large molecules and consequently have high molecular masses. Poor water solubility is a major difficulty in the process of drug formulation. ${ }^{39}$ Erythromycin estolate and azithromycin dihydrate (the salts used in this study) are both particularly insoluble in water. A study conducted by Nakornchai and Konthiang ${ }^{14}$ concluded that erythromycin inhibited growth of P. falciparum at an $\mathrm{IC}_{50}$ value of $58.2 \pm 7.0 \mu \mathrm{M}$ and azithromycin at $8.4 \pm 1.2 \mu \mathrm{M}$. An $\mathrm{IC}_{50}$ value depicts the concentration of the drug needed for $50 \%$ of the maximum inhibitory effect in a test system. ${ }^{40}$ Formulation design in this study was based on concentration ranges of $0-80 \mu \mathrm{M}$ for erythromycin and $0-8 \mu \mathrm{M}$ for azithromycin. The high molecular masses, relatively high $\mathrm{IC}_{50}$ values and both drugs' poor solubility proved a challenge in the formulation process. Previous studies indicated that co-formulating lipid soluble drugs with the oil phase during manufacturing proved successful. ${ }^{35}$ The $4 \%$ Pheroid vesicle formulation containing azithromycin appeared to have small Pheroid vesicles $(1.21 \pm 0.11 \mu \mathrm{m})$ with large crystal aggregates (Figure 1a). Increasing the oil phase concentration to $8 \%$ did not improve the formulation and large crystals were still observed with large mean particle size of $202.42 \pm 0.74 \mu \mathrm{m}$ (Figures $1 \mathrm{~b}$ and $\mathrm{d}$ ). Entrapping azithromycin in the diluted Pheroid vesicles led to more uniform particle sizes (Table 1) and decreased the particle size significantly $(P<0.05)$, with no visible crystal formation (Figure 1c). The formulations containing erythromycin and 
azithromycin entrapped in Pheroid vesicles were compared with the water soluble antibiotics doxycycline and tetracycline entrapped in Pheroid vesicles. The particles of azithromycin and erythromycin were significantly larger $(P<0.05)$ than the Pheroid vesicles control, tetracycline and doxycycline (Table 1). Micro scale formulations of azithromycin $(\sim 200 \mu \mathrm{m})$ have been developed containing microspheres of high dose azithromycin in a powder form for oral suspension. The formulation were evaluated clinically and provided complete release within $3 \mathrm{~h}$, good tolerability without decreased bioavailability. ${ }^{41}$ A similar formulation could be developed with Pheroid vesicles that could prove successful for malaria treatment.

\section{HRP II method validation}

In order to assess the performance of the HRP II ELISA assay for in vitro drug sensitivity testing, $P$. falciparum were diluted to a range of parasitemia $(0.25-4 \%)$. The results are shown in Figure 2 in terms of absorbance at $450 \mathrm{~nm}$ (Figure 2a) and the amount of HRP II $\left(\mathrm{ng} \mathrm{ml}^{-1}\right.$ ) calculated from the standard curve. The assay proved to be sensitive at all parasitemia tested as indicated by the linearity of the fitted curve. This differed from results were obtained by Kifude et al., ${ }^{42}$ who demonstrated that the lower level of quantification; that is, the point at which the analysis is feasible, was $3.19 \mathrm{ng} \mathrm{ml}^{-1}$, corresponding to an OD of 0.05 . Linearity in our assay was obtained at all parasitemia tested and the lower limit of quantification was $2.18 \mathrm{ng} \mathrm{ml}^{-1}$ corresponding to an OD of 0.04 . These results confirm the results found by Noedl et al., ${ }^{43}$ that the assay can detect low levels of parasitemia and have a wide dynamic range. The performance of the assay was also assessed for the Pheroid vesicles. The formulations are opaque white in the concentrated form. After dilution the color of the formulation was clearer but some opacity remains. The

Table 1 Particle size and span of Pheroid vesicles control and Pheroid vesicles (4\%) entrapping erythromycin, azithromycin, doxycycline and tetracycline

\begin{tabular}{lcc}
\hline & \multicolumn{2}{c}{ Particle size $(\mu \mathrm{m})$} \\
\cline { 2 - 3 } \multicolumn{1}{c}{ Parameters } & Mean & Span \\
\hline Pheroid vesicles & $2.19 \pm 0.24$ & $2.49 \pm 0.08$ \\
Pheroid azithromycin & $72.76 \pm 10.73^{\mathrm{a}}$ & $2.20 \pm 0.60$ \\
Pheroid erythromycin & $100.62 \pm 29.27^{\mathrm{a}}$ & $2.53 \pm 0.49$ \\
Pheroid doxycycline & $1.10 \pm 0.34$ & $4.46 \pm 0.54$ \\
Pheroid tetracycline & $8.1 \pm 0.61$ & $4.65 \pm 0.61$ \\
\hline
\end{tabular}

Results are expressed as mean \pm s.e.m. $(n=6)$.

a Statistical significant differences compared with Pheroid vesicles $(P \leqslant 0.05)$.

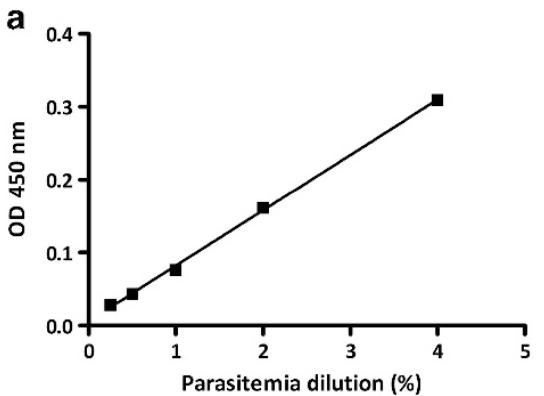

Figure 2 Relationship of the absorbance (a) and HRP II levels (b) to P. falciparum percentage dilution. Results are expressed as mean \pm s.e.m ( $n=6$ ).

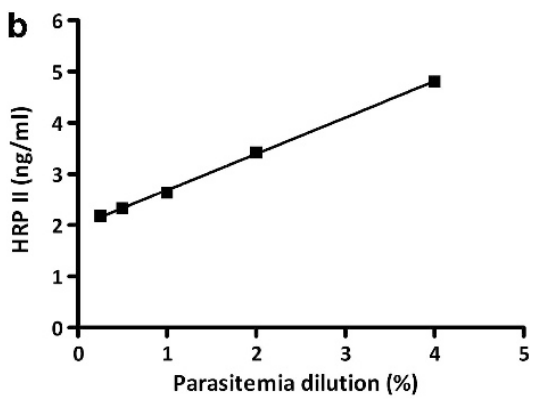

formulations were evaluated for interference with the assay in terms of background fluorescence that may be caused by the formulations. The assay was also evaluated in terms of whether the long incubation times of the planned experiments would influence the assay. The samples containing Pheroid vesicles had an average background absorbance value of $0.025 \pm 0.006(n=12)$. Biological threshold are calculated by adding 3SD to the average background level, which gave a value of 0.031 ; that is, close to the lower limit of quantification. The data were normalized in terms of the Pheroid background to exclude possible interferences of the data.

\section{Drug efficacy assays}

The life cycle of $P$. falciparum inside the red blood cells progress from inert rings to larger metabolically active trophozoites and then to schizonts that undergoes cellular division and release merozoites. The whole cycle last $48 \mathrm{~h}$ and it has been found that tested macrolides exert a delayed effect after $96 \mathrm{~h}$ in the third generation rings. ${ }^{44}$ In this study, the drugs alone and entrapped in Pheroid vesicles were incubated with $P$. falciparum for 48, 96 and $144 \mathrm{~h}$ (Table 2). The effect of erythromycin was more pronounced after $96 \mathrm{~h}$ but did not markedly decrease after $144 \mathrm{~h}$. The $\mathrm{IC}_{50}$ of azithromycin decreased at $96 \mathrm{~h}$ and further decreased at $144 \mathrm{~h}$. The Pheroid vesicle formulation entrapping the drugs had significantly lower $\mathrm{IC}_{50}$ values compared to the drugs alone. Azithromycin and erythromycin are slow acting antimalarials and are proven to be more potent against cultured $P$. falciparum after two life cycles after the start of treatment. ${ }^{16,26}$ Formulating the antibiotics with Pheroid vesicles improved the efficacy of the antibiotics at all the time points. This could possibly be attributed to increased solubilization of the poorly water soluble drugs. Previous studies with poorly water soluble artemisinin derivative artemisone showed similar results, where the Pheroid

Table 2 IC $_{50}$ values of azithromycin and erythromycin (controls) and both Pheroid vesicles entrapping erythromycin and azithromycin at 48,96 and $144 \mathrm{~h}$ incubation with $P$. falciparum

\begin{tabular}{lccc}
\hline & \multicolumn{3}{c}{$I C_{50}(\mu M)$} \\
\hline Drug and formulation & $48 \mathrm{~h}$ & $96 \mathrm{~h}$ & $144 \mathrm{~h}$ \\
Erythromycin & $53.85 \pm 0.34$ & $12.61 \pm 0.50^{\mathrm{a}}$ & $12.49 \pm 0.25^{\mathrm{a}}$ \\
Pheroid erythromycin & $15.14 \pm 1.17^{\mathrm{b}}$ & $4.43 \pm 0.82^{\mathrm{b}}$ & $4.09 \pm 3.3^{\mathrm{b}}$ \\
Azithromycin & $23.90 \pm 0.25$ & $3.88 \pm 0.50$ & $2.69 \pm 0.43^{\mathrm{a}}$ \\
Pheroid azithromycin & $9.27 \pm 0.52^{\mathrm{b}}$ & $0.40 \pm 0.20^{\mathrm{b}}$ & $0.38 \pm 0.21^{\mathrm{a}, \mathrm{b}}$ \\
\hline
\end{tabular}

Results are expressed as mean \pm s.e.m. $(n=6)$.

astatistical significant differences relative to $48 \mathrm{~h}$

bifferences relative to the respective drug control. 
Table $3 \quad \mathrm{IC}_{50}$ values of standard antimalarial drugs, antibiotics and triclosan entrapped in Pheroid vesicles after $96 \mathrm{~h}$ incubation time

\begin{tabular}{lrc}
\hline & \multicolumn{2}{c}{$I_{50}(\mu \mathrm{m})$} \\
\cline { 2 - 3 } \multicolumn{1}{c}{ Drug } & \multicolumn{1}{c}{ Control } & Pheroid $^{\mathrm{Im}}$ vesicles $^{\circ}$ \\
\hline Chloroquine & $0.23 \pm 0.02$ & $0.15 \pm 0.05$ \\
Quinine & $15.58 \pm 0.14$ & $7.1 \pm 0.07^{\mathrm{a}}$ \\
Doxycycline & $19.84 \pm 0.02$ & $14.82 \pm 0.04$ \\
Tetracycline & $6.09 \pm 0.12$ & $5.45 \pm 0.07$ \\
Erythromycin & $13.85 \pm 0.34$ & $3.14 \pm 1.17^{\mathrm{a}}$ \\
Azithromycin & $3.90 \pm 0.25$ & $0.27 \pm 0.52^{\mathrm{a}}$ \\
Triclosan & $0.31 \pm 0.06$ & $0.18 \pm 0.09^{\mathrm{a}}$ \\
\hline
\end{tabular}

Results are expressed mean \pm s.e.m. $(n=6)$

astatistically significant differences compared with the control.

vesicle formulation significantly increased the bioavailability of artemisone 4.5 times. $^{36}$

The $\mathrm{IC}_{50}$ values of erythromycin and azithromycin entrapped in Pheroid vesicles were compared with other antibiotics and antimalarial drugs entrapped in Pheroid vesicles in a separate experiment (Table 3). Previous studies have indicated $\mathrm{IC}_{50}$ values of $220 \mathrm{~nm}$ of chloroquine against RSA $11^{45}$ and $169.8 \mathrm{~nm}$ against K1. ${ }^{46}$ Chloroquine is investigated for used in combination with azithromycin in intermittent preventative treatment during pregnancy (iPTp) and the two drugs are synergistic. Pheroid vesicles decreased the $\mathrm{IC}_{50}$ of both drugs and a formulation combining the two drugs could prove successful in malaria treatment. Quinine is mostly used in combination with tetracycline, doxycycline or clindamycin as a second line treatment. ${ }^{47} \mathrm{~A}$ twofold decrease of $\mathrm{IC}_{50}$ of quinine was seen in combination with Pheroid vesicles. This decrease was statistically significant. The decrease in $\mathrm{IC}_{50}$ values with tetracycline and doxycycline was not as pronounced as for azithromycin and erythromycin. Doxycycline also showed delayed effects after $96 \mathrm{~h}$ similar to azithromycin. ${ }^{44}$ These antibiotics are used in combination with standard antimalarial drugs. Doxycycline is more hydrophobic than tetracycline, ${ }^{48}$ which could explain the more pronounced decrease in $\mathrm{IC}_{50}$ values observed by entrapping the drug in Pheroid vesicles. A recent study indicated that the $\mathrm{IC}_{50}$ values of azithromycin and doxycycline lies between $1-10 \mu \mathrm{m}$ with no major difference between two drug sensitive strains (NF54 and D6) and five drug resistant strains (K1, W2, 7G8, TM90C2A and V1/S). These strains are all from different regions with different sensitivities to various drugs. Relative to drugs such as artemisinin derivatives artesunate and artemisone and the new synthesized compounds OZ277 and OZ439 (nanomolar range) the antibiotics have relatively high $\mathrm{IC}_{50}$ values. ${ }^{49}$ The $\mathrm{IC}_{50}$ values from this recent study correlates well with the results from our study against RSA 11.

The antimicrobial biocide 5-chloro-2-(2,4-dichlorophenoxy) phenol, commonly known as triclosan, targets the enoyl-acyl carrier protein (ACP) reductase of the type II fatty acid biosynthesis pathway. Triclosan inhibits $P$. falciparum in a stage specific manner, preventing trophozoites from invading erythrocytes after $48 \mathrm{~h} .{ }^{50}$ Triclosan has been reported to inhibit the growth of drug-resistant and drugsensitive $P$. falciparum in vitro whole cell assays with $\mathrm{IC}_{50}$ values of $0.2-0.9 \mu \mathrm{M} .{ }^{51}$ This activity was significantly better than that of the antibiotic thiolactomycin, which is thought to act on FAS-II enzymes including ketoacyl-ACP synthase, showed an $\mathrm{IC}_{50}$ value of $\sim 50 \mu \mathrm{M}$ against $P$. falciparum. ${ }^{52}$ Triclosan was also reported to be effective in clearing Plasmodium berghei from mice with a single s.c. $38 \mathrm{mg} \mathrm{kg}^{-1}$ injection of triclosan, with no apparent toxic side effects. ${ }^{50}$ The discovery of the apicoplast in Plasmodium has offered a promising new drug target. Pheroid vesicles significantly decreased the $\mathrm{IC}_{50}$ of triclosan. In comparison with other antibiotics triclosan has a low $\mathrm{IC}_{50}$ in vitro; however, a recent correspondence indicated discrepancies in the efficacy of triclosan in vivo. ${ }^{53}$

This study describes the in vitro efficacy of Pheroid vesicles containing antibiotics against malaria. Entrapping the drugs in Pheroid vesicles decreased the $\mathrm{IC}_{50}$ values of the antibiotics with varied levels of significance. Azithromycin and erythromycin were successfully formulated within the Pheroid vesicles and micro vesicle formulations were observed. The Pheroid vesicles containing azithromycin and erythromycin enhanced the activity of the drugs against a chloroquine-resistant strain of $P$. falciparum. A more pronounced delayed effect against $P$. falciparum was observed after treatment by azithromycin and erythromycin entrapped Pheroid vesicles. Pheroid vesicles also proved successful in decreasing the $\mathrm{IC}_{50}$ values of chloroquine, quinine and triclosan. The formulations with chloroquine, azithromycin and erythromycin show great promise in the treatment of malaria and possibly for use in iPTp. However, the increase of in vitro efficacy should be confirmed in vivo and the pharmacokinetics of the Pheroid vesicles containing antibiotics should be determined, before a product can be considered.

1 WHO. World Malaria Report 2011 (WHO, Geneva, Switzerland, 2011).

2 Cox, F. E. G. Modern Parasitology, A textbook of Parasitology 14-18 (Blackwell Science, Cambridge, 1996).

3 Agnandji, S. T. First results of Phase 3 trial of RTS,S/ASO1 malaria vaccine in African children. N. Engl. J. Med. 365, 1863-1875 (2011).

4 O'Brien, C., Henrich, P. P., Passi, N. \& Fidock, D. A. Recent clinical and molecular insights into emerging artemisinin resistance in Plasmodium falciparum. Curr. Opin. Infect. Dis. 24, 570-577 (2011).

5 Pešić, D. et al. Design, synthesis, and in vitro activity of novel 2'-0-substituted 15-membered azalides. J. Med. Chem. 55, 3216-3227 (2012).

6 Perić, M. et al. Antimalarial activity of 9A-N- substituted 15-membered azalides with improved in vitro and in vivo activity over azithromycin. J. Med. Chem. 55, 1389-1401 (2012).

7 Praveen Kumar, S. et al. Design, synthesis and evaluation of 3-methylene-substituted indolinones as antimalarials. Eur. J. Med. Chem. 46, 927-983 (2011).

8 Glória, P. M. C. et al. Aza vinyl sulfones: Synthesis and evaluation as antiplasmodial agents. Biorganic Med. Chem. 19, 7635-7642 (2011).

9 Banerjee, A. K., Arora, N. \& Murty, U. S. N. Analysing a potential drug target $\mathrm{N}$-myristoyltransferase of Plasmodium falciparum trough in silico approaches. J. Glob. Infect. Dis. 4, 43-54 (2012).

10 Qidwai, T. \& Khan, F. Antimalarial drugs and drug targets specific to fatty acid metabolic pathway of Plasmodium falciparum. Chem. Biol. Drug. Des 80, 155-172 (2012).

11 Kuntworbe, N. \& Al-Kassas, R. Design and in vitro haemolytic evaluation of cryptolepine hydrochloride-loaded gelatin nanoparticles as a novel approach for the treatment of malaria. AAPS Pharm. Sci. Tech. 13, 568-581 (2012).

12 Slabbert, C., du Plessis, L. H. \& Kotzé, A. F. Evaluation of the physical properties and stability of two lipid drug delivery systems containing mefloquine. Int. J. Pharm. 409, 209-215 (2011).

13 Isacchi, B. et al. Artemisinin and artemisinin plus curcumin liposomal formulations: Enhanced antimalarial efficacy against Plasmodium berghei-infected mice. Eur. J. Pharm. Biopharm. 80, 528-534 (2012).

14 Nakornchai, S. \& Konthiang, P. Activity of azithromycin or erythromycin in combination with antimalarial drugs against multidrug-resistant Plasmodium Falciparum in vitro. Acta Trop. 100, 185-191 (2006).

15 Pradines, B. et al. Antibiotics for prophylaxis of Plasmodium falciparum infections: in vitro activity against doxycycline against Senegalese insolates. Am. J. Trop. Med. Hyg. 62, 82-85 (2000).

16 Pradines, B. et al. In vitro activities of antibiotics against Plasmodium falciparum are inhibited by iron. Antimicrob. Agents Chemother. 45, 1746-1750 (2001).

17 Sood, S. K. Macrolides: clarithromycin and azithromycin. Semin. Pediatric Infect Diseases 10, 23-30 (1999)

18 Chambers, H. F. in Basic and Clinical Pharmacology. 9th edn. (Katzung, B. G.) 754-763 (McGraw-Hill, New York, 2004).

19 Noedl, H. et al. Antimalarial activity of azithromycin, artemisinin and dihydroartemisinin in fresh isolates of Plamsodium falciparum in Thailand. Acta Trop. 80, 39-44 (2001).

20 Gingras, B. A. \& Jensen, J. B. Activity of azithromycin (CP-62,993) and erythromycin against chloroquine-sensitive and chloroquine-resistant strains of Plasmodium falciparum in vitro. Am. J. Trop. Med. Hyg. 47, 378-382 (1992). 
21 Gingras, B. A. \& Jensen, J. B. Antimalarial activity of azithromycin and erythromycin against Plasmodium berghei. Am. J. Trop. Med. Hyg. 49, 101-105 (1993).

22 Menezes, C. M. S. et al. In vitro evaluation of erythromycin in chloroquine-resistant brazilian P. falciparum freshly isolates: modulating effect and antimalarial activity evidence. Rev. I Med. Trop. 41, 249-253 (1999).

23 Pang, L. W. et al. Failure of large-dose erythromycin in combination with a standard dose of chloroquine or quinine in the treatment of human falciparum malaria. B. World Health Organ. 63, 739-743 (1985).

24 Sidhu, A. B. S. et al. In vitro efficacy, resistance selection, and structural modelling studies implicate the malarial parasite apicoplast as the target of azithromycin. J. Biol. Chem. 282, 2494-2504 (2006).

25 Miller, R. S. et al. Effective treatment of uncomplicated Plasmodium falciparum malaria with azithromycin-quinine combinations: a randomized, dose-ranging study. Am. Soc. Trop. Med. Hyg. 74, 401-406 (2006).

26 Yeo, A. E. \& Rieckman, K. H. Prolonged exposure of Plasmodium falciparum to ciprofloxacin increases anti-malarial activity. J. Parasitol. 80, 158-160 (1994).

27 Vallely, A., Vallely, L., Changalucha, J., Greenwood, B. \& Chandramohan, D. Intermittent preventive treatment for malaria in pregnancy in Africa: what's new, what's needed? Malaria J. 6, 1-13 (2007).

28 Ohrt, C., Willingmyre, G. D., Lee, P., Knirsch, C. \& Milhous, W. Assessment of azithromycin in combination with other antimalarial drugs against Plasmodium falciparum in vitro. Antimicrob. Agents Chemother. 46, 2518-2524 (2002).

29 Anderson, S. L. et al. Efficacy of azithromycin as a causal prophylactic agent against murine malaria. Antimicrob. Agents Chemother. 38, 1862-1863 (1994).

30 Taylor, W. R. et al. Tolerability of azithromycin as malaria prophylaxis in adults in North East Papua, Indonesia. Antimicrob. Agents Chemother. 47, 2199-2203 (2003).

31 Andersen, S. L. et al. Successful doubleblinded, randomized, placebo-controlled field trial of azithromycin and doxycycline as prophylaxis for malaria in western Kenya. Clin. Infect. Dis. 26, 146-150 (1998).

32 Krudsood, S. et al. A randomised clinical trial of combination artesunate and azithromycin for treatment of uncomplicated Plasmodium falciparum malaria in Thailand. Southeast Asian J. Trop. Med. Public Health. 31, 801-807 (2000).

33 Chico, R. M., Pittrof, R., Greenwood, B. \& Chandramohan, D. Azithromycin-chloroquine and the intermittent preventive treatment of malaria in pregnancy. Malaria J. 7, 255-269 (2008).

34 Du Plessis, L. H., Lubbe, J., Strauss, T. \& Kotzé., A. F. Enhancement of nasal and intestinal calcitonin delivery by the novel Pheroid ${ }^{\mathrm{TM}}$ fatty acid based delivery system, and by N-trimethyl chitosan chloride. Int. J. Pharm. 385, 181-186 (2010).

35 Steyn, J. D. et al. Absorption of the novel artemisinin derivatives artemisone and artemiside: Potential application of Pheroid ${ }^{\mathrm{TM}}$ technology. Int. J. Pharm. 414 260-266 (2011).

36 Thipine, M., Letscher-Bru, V. \& Herbrecht, R. Amphoterecin B and its new formulations: pharmacologic characteristics, clinical efficacy, and tolerability. Transpl. Infect. Dis. 1999, 273-283 (1999).
37 Oh, Y.-K., Nix, D. E. \& Straubinger, R. M. Formulation and efficacy of liposome encapsulated antibiotics for therapy of intracellular Mycobacterium avium infection. Antimcrob. Agents Chemother. 39, 2104-2111 (1995).

38 Trager, W. \& Jensen, J. B. Human malaria parasites in continuous culture. Science 193, 673-675 (1976).

39 Chingunpituk, J. Nanosuspension technology for drug delivery. Walailak J. Sci. Tech. 4 139-153.

40 Stepniewska, K., Chotivanich, K., Brockman, A., Day, N. P. J. \& White, N. J. Overestimating resistance in field testing of malaria parasites: simple methods for estimating high EC50 values using a Bayesian approach. Malaria J. 6, 4-13 (2007).

41 Lo, J. B., Appel, L. E., Herbig, S. M., McCray, S. B. \& Thombre, A. G. Formulation design and pharmaceutical development of a novel controlled release form of azithromycin for single-dose therapy. Drug. Dev. Indus. Pharm. 35, 1522-1529 (2009).

42 Kifude, C. M. et al. Enzyme-linked immunosorbent assay for detection of Plasmodium falciparum histidine-rich protein 2 in blood, plasma and serum. Am. Soc. Microbiol. 15, 1012-1018 (2008).

43 Noedl, H., Wongsrichanalai, C. \& Wernsdorfer, W. H. Malaria drug-sensitivity testing: new assays, new perspectives. Trends Parasitol. 19, 175-181 (2003).

44 Dahl, E. L. \& Rosenthal, P. J. Multiple antibiotics exert delayed effects against the Plasmodium falciparum apicoplast. Antimicrob. Agents Chemother. 51, 3485-3490 (2007).

45 Fivelman, Q. L., Walden, J. C., Smith, P. J., Folb, P. I. \& Barnes, K. I. The effect of artesunate combined with standard chloroquine-sensitive and chloroquine-resistant strains of Plasmodium falciparum in vitro. Trans. 93, 429-432 (1999).

46 Wu, C.-P., Van Schalwyk, D. A., Taylor, D., Smith, P. J. \& Chibale, K. Reversal of chloroquine resistance in Plasmodium falciparum by $9 \mathrm{H}$-xanthine derivatives. Int. J. Antimicrob. Agents 26, 170-175 (2005).

47 Achan, J. et al. Quinine, an old anti-malarial drug in a modern world: role in the treatment of malaria. Malaria J. 10, 144 (2011).

48 Agwuh, K. N. \& MacGowan, A. Pharmacokinetics and pharmacodynamics of the tetracyclines including glycylcyclines. J. Antimcrob. Chemother. 58, 256-265 (2006).

49 Delves, M. et al. The activities of current antimalarial drugs on the life cycles of Plasmodium: A comparative study with human and rodent parasites. PLOS Med. 9, e1001169 (2012).

50 Surolia, N. \& Surolia, A. Triclosan offers protection against blood stages of malaria by inhibiting enoyl-ACP reductase of Plasmodium falciparum. Nature Med. 7, 167-173 (2001).

$51 \mathrm{Kuo}, \mathrm{M}$. R. Targeting tuberculosis and malaria through inhibition of enoyl reductase. J. Biol. Chem. 23, 20851-20859 (2003).

52 Waller, R. F. et al. Nuclera-encoded proteins target to the plastid in Toxoplasma gondi and Plasmodium falciparum. Proc. Natl Acad. Sci. USA 95, 12352-12357 (1998).

53 Baschong, W. et al. Triclosan is minimally effective in rodent malaria models. Nature Med. 17, 33-34 (2011). 